\title{
TRANSCRIPTION ACTIVATOR-LIKE EFFECTOR NUCLEASES-AMAZING TOOL FOR GENOME EDITING
}

\author{
${ }^{1}$ Avantika Singh and ${ }^{2}$ Vivek Pratap Singh \\ ${ }^{1}$ University School of Biotechnology, Guru Gobind Singh Indraprastha University, New Delhi, India \\ ${ }^{2}$ Department of Surgery, Baylor College of Medicine, One Baylor Plaza, Houston, Texas-77030, USA
}

Received 2013-07-29, Revised 2013-08-09; Accepted 2013-08-19

\begin{abstract}
Transcription Activator-Like Effector Nucleases (TALENs) are used to cut specific target DNA sequence in order to knock out a gene or to change its sequence. They are formed by the fusion of TALE protein serving as DNA binding domain along with non-specific DNA cleaving nucleases. Its specificity towards DNA bases in the target sequence is derived from the structure of the DNA binding domain, consisting of variable pair of amino acids in middle of the tandem array of repeated segment. Earlier targeted genome editing was performed using Zinc Finger Nucleases (ZFN). Recently TALENS have rapidly emerged as alternative to Zinc Finger Nucleases for genome editing and introducing targeted Double Stranded Break. The TALEN approach improves on tools currently available for genome modification. This breakthrough could eventually make it possible to efficiently modify plant, animal and even human genomes. Targeted nucleases offer the potential to correct or disrupt the gene product or sequences that causes the disorder and thus motivates the strategies for treatment of wide range of genetic and other diseases. The road to practical use of TALENs could still contain potholes. But particularly to academics, the potential of TALENs seems limitless.
\end{abstract}

Keywords: TALENs, TALE Proteins, Zinc Finger Nucleases, Genome Modification

\section{INTRODUCTION}

\subsection{TALENS}

Newly developed engineered nuclease Transcription Activator-Like Effector Nucleases (TALENs) can efficiently modify any sequence of interest in living cells or organisms. It can make replacement and geneeditingtherapies, Double-strand DNA breaks at specific sites in living cells, possibly leading to better gene. TALENs are hybrid protein containing a DNA binding domain and nonspecific fokl nuclease domain. DNA binding domain is derived from TALE protein secreted by a plant pathogen- Xanthomonasspp that attacks more than 350 plant species and causes diseases such as citrus canker and black rot (Kay and Bonas, 2009). TALE protein from bacteria alters the gene transcription in host plant (Kya et al., 2007). The DNA binding domain binds specifically to the target sequence to be cleaved and the nuclease introduce double-stand breaks, thus providing an efficient tool for targeted genome engineering (Christian et al., 2010; Boch et al., 2009).

\subsection{Structure of TALENs}

The fundamental building block of DNA binding region of TALENs i.e., TALE protein consists of multiple sets of highly conserved repeat domain of same or almost same 34 amino acids occurring in tandem (Schornack et al., 2006; Ackerveken et al., 1996; Zhu et al., 1998). As shown in the Fig. 1, each repeat targets a specific base of DNA, identity of which is determined by a hyper variable pair of amino acid typically found at position 13 and 14 of the repeat. Each of these pair of amino acids binds to specific nucleotide in the target DNA (Boch et al., 2009; Moscou and Bogdanove, 2009; Herbers et al., 1992; Yang et al., 2005). Rest of the 34 amino acids in the repeats is nearly always the same. Thus the overall number of repeats matches an equal number of bases. In 2010, first nucleases were attached to TALE proteins for the purpose of genome engineering.

Corresponding Author: Vivek Pratap Singh, Department of Surgery, Baylor College of Medicine, One Baylor Plaza, Houston, Texas-77030, USA 


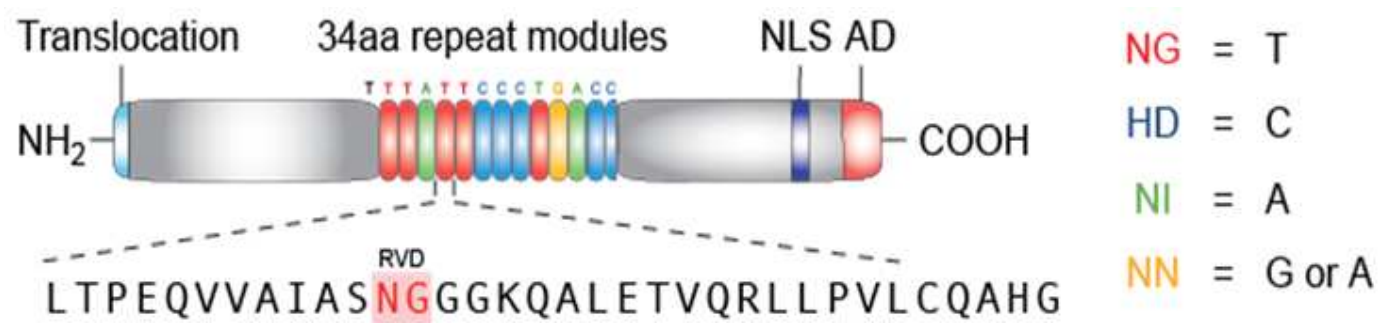

Fig. 1. Structure of TALENs

\subsection{Similarity with Zinc Finger Nucleases}

Zinc fingers are finger like sections of certain DNA binding proteins found in mammals (Elrod-Erickson et al., 1996; Isalan et al., 1997). They home in on specific target sequences of DNA. A different Zinc finger recognizes different sets of three bases (Bibikova et al., 2003). When combined with nucleases, they can knock out genes and also introduce new versions of genes at specific location in genome (Urnov et al., 2005; Wood et al., 2011). Several types of genomic alterations can be introduced with ZFNs, including point mutations, deletions, insertions, inversions, duplications and translocations, thus providing researchers with unprecedented tools to perform genetic manipulations (Wood et al., 2011; Townsend et al., 2009).

\subsection{Problems with ZFN}

ZFN are difficult to design and are expensive. TALENs could provide a cheaper alternative. As TALENs have high rate of cleavage activity and limitless targeting range, genome engineering using TALENs was named the 2011 method of the year by nature publishing group. Almost everybody who has once worked on ZFN is now shifting to TALENs, not only because of ease of making TALENs (Boch et al., 2009; Moscou and Bogdanove, 2009) but unlike ZFN, Sangamo Gregory doesn't have a lock on TALENs. Sangamo controls almost all of the IPR to ZF technology, which is the main reason for the high cost of ZFN. But no patents have been issued yet in the field of TALENs.

\subsection{Applications}

Researchers using TALENs have engineered genomes in yeast, nematodes, tobacco, Arabidopsis, fruitfly, roundworm, cricket, zebrafish, frog, rat, pig, cow, thale cress, rice and silkworm. TALENs have also been used to introduce specific insertions in human somatic and pluripotent stem cells using double-stranded donor templates.

\subsection{Gene Therapy}

In contrast to therapies that treat symptoms of genetic diseases, the ability to target essentially any DNA sequence with TALENs will undoubtedly motivate the exploration of both gene-correction and gene-disruption strategies for the treatment of a wide range of genetic and other diseases (Miller et al., 2010). TALENs can correct the genetic defect underlying sickle cell disease, $\alpha 1$ antitrypsin disease and Parkinson's disease. TALENs can also go into a cell and turn on a specific gene, giving finer control over gene expression (Joung and Sander, 2013).

\subsection{Livestock}

TALENs have also been a welcome addition to the toolbox of those seeking to genetically modified pigs, cows and livestock to make versions that are more useful for biomedical research or food production. Precision crossbreeding can be done using TALENs that can greatly speed up the introduction of new traits to livestock. TALENs have been used to inactivate the gene encoding Low-Density Lipoprotein (LDL) receptor in pigs, thereby generating a model for familial hypercholesterolemia (Carlson et al., 2012).

\subsection{Agriculture}

Nuclease-mediated editing of agricultural plants having long reproductive cycles may greatly decrease the time required to generate new agriculturally-relevant varieties compared with traditional breeding strategies. To date, TALENs have been used to introduce knockout mutations in Arabidopsis thaliana (Morbitzer et al., 2010) and to confer resistance to infection by Xanthomonas bacteria in rice by disrupting the target sites of naturally occurring TALEs that contribute to pathogenicity.

\subsection{Miscellaneous}

Targeted insertions could be used to fuse endogenous genes to genes encoding fluorescent proteins or epitope 
tags to visualize protein expression, distribution and interactions. In addition to the generation of such fusions, HDR-based approaches might be used to create isogenic human or other mammalian cell lines bearing specific Single Nucleotide Polymorphisms (SNPs), thereby potentially enabling studies to determine the functional significance of these sequence variants (Geißler et al., 2011).

\subsection{Limitations}

TALENs are bigger and bulkier than zinc finger nucleases, so getting them to their target DNA may sometimes be tricky. Also the repetitive nature of TALEN means that the gene encoding it, once delivered into the cell, may be more susceptible to DNA rearrangements-where the nuclease cut the unintended DNA sequence.

\section{CONCLUSION}

Formation of effective genome editing tools is requisite for fundamental research, genetic engineering and gene therapy. Efficient construction and application of Transcription Activator-Like Effector Nucleases (TALENs) in several organisms precede an exciting new era for genome editing. TALENs have already begun to revolutionize research and medicine and their potential over the coming years seems enormous, basic biologists can study molecular pathway gene-by-gene, protein by protein. Plant biologists will be able to introduce multiple traits at once. It will just become a tool that every molecular biologist has in the lab. The optimization of methods for efficiently delivering TALENs or nucleic acids encoding them into cells will also be an important area for future research. Another potential area for future exploration will be the creation of fusion proteins that harbor domains others than nucleases.

\section{ACKNOWLEDGEMENT}

I wish to thank Professor K. Kannan (University School of Biotechnology) for clarification of some aspects of the molecular biology of targeted nucleases as well as for his valuable comments.

\section{REFERENCES}

Ackerveken, G.V.D., E. Marois and U. Bonas, 1996. Recognition of the bacterial avirulence protein avrbs3 occurs inside the host plant cell. Cell, 87: 1307-1316. DOI: 10.1016/S0092-8674(00)81825-5
Bibikova, M., K. Beumer, JK. Trautman and D. Carroll, 2003. Enhancing gene targeting with designed zinc finger nucleases. Science, 300: 764-764. DOI: 10.1126/science.1079512

Boch, J., H. Scholze, S. Schornack, A. Landgraf and S. Hahn et al., 2009. Breaking the code of DNA binding specificity of TAL-type III effectors. Science, 326: 1509-1512. DOI: 10.1126/science. 1178811

Christian, M., T. Cermak, E.L. Doyle, C. Schmidt and F. Zhang et al., 2010. Targeting DNA double-strand breaks with TAL effector nucleases. Genetics, 186: 757-761. DOI: 10.1534/genetics.110.120717

Carlson, D.F., W. Tan,, G.S. Lillico, D. Stverakova and C. Proudfoot et al., 2012. Efficient TALENmediated gene knockout in livestock. Proceedings of the National Academy of Sciences, (NAS' 12), pp: 17382-17387. DOI: 10.1073/pnas.1211446109

Elrod-Erickson, M., M.A. Rould, L. Nekludova and C.O. Pabo, 1996. Zif268 protein-DNA complex refined at 1.6å: A model system for understanding zinc fingerDNA interactions. Structure, 4: 1171-1180. DOI: 10.1016/S0969-2126(96)00125-6

Geißler, R., H. Scholze, S. Hahn, J. Streubel and U. Bonas et al., 2011. Transcriptional activators of human genes with programmable dna-specificity. PLoS ONE, 6: e19509-e19509. DOI: 10.1371/journal.pone.0019509, PMID: 21625585

Herbers, K., J. Conrads-Strauch and U. Bonas, 1992. Race-specificity of plant resistance to bacterial spot disease determined by repetitive motifs in a bacterial avirulence protein. Nature, 356: 172-174. DOI: 10.1038/356172a0

Isalan, M., Y. Choo and A. Klug, 1997. Synergy between adjacent zinc fingers in sequence-specific DNA recognition. Proc. Natl. Acad. Sci. USA., 94: 5617-5621. DOI: 10.1073/pnas.94.11.5617

Joung, J.K. and J.D. Sander, 2013. TALENs: A widely applicable technology for targeted genome editing. Nature Rev. Molecul. Cell Biol., 14: 49-55. DOI: $10.1038 / \mathrm{nrm} 3486$

Kay, S. and U. Bonas, 2009. How Xanthomonas type III effectors manipulate the host plant. Curr. Opin. Microbiol., 12: 37. DOI: 10.1016/j.mib.2008.12.006

Kya, S., S. Hahn, E. Marous, G. Hause and Ulla Bonas, 2007. A bacterial effector acts as a plant transcription factor and induces a cell size regulator. Science, 318: 648-651. DOI: 10.1126/science. 1144956 
Miller, J.C., S. Tan, G. Qiao, K.A. Barlow and J. Wang et al., 2010. A tale nuclease architecture for efficient genome editing. Nature Biotechnol., 29: 143-148. DOI: $10.1038 /$ nbt. 1755

Morbitzer, R., P. Romer, J. Boch and T. Lahaye, 2010. Regulation of selected genome loci using de novoengineered Transcription Activator-Like Effector (TALE)-type transcription factors. Proc. Natl. Acad. Sci., 107: 21617-21622.

DOI: 10.1073/pnas.1013133107

Moscou, M.J. and A.J. Bogdanove, 2009. A simple cipher governs DNA recognition by TAL effectors. Science, 326: 1501-1501. DOI: 10.1126/science. 1178817

Schornack, S., A. Meyer, P. Römer, T. Jordan and T. Lahaye, 2006. Gene-for-gene-mediated recognition of nuclear-targeted AvrBs3-like bacterial effector proteins. J. Plant Physiol., 163: 256-272. DOI: 10.1016/j.jplph.2005.12.001

Townsend, J.A., D.A. Wright, R.J. Winfrey, F. Fu and M.L. Maeder et al., 2009. High-frequency modification of plant genes using engineered zincfinger nucleases. Nature, 459: 442-445. DOI: 10.1038 /nature 07845
Urnov, F.D., J.C. Miller, Y.L. Lee, C.M. Beausejour and J.M. Rock et al., 2005. Highly efficient endogenous human gene correction using designed zinc-finger nucleases. Nature, 435: 646-651. DOI: 10.1038/nature03556

Wood, A.J., T.W. Lo, B. Zeitler, C.S. Pickle and E.J. Ralston et al., 2011. Targeted genome editing across species using ZFNs and TALENs. Science, 333: 307-307. DOI: 10.1126/science. 1207773

Yang, B., A. Sugio and F.F. White, 2005. Avoidance of Host Recognition by Alterations in the Repetitive and C-Terminal Regions of AvrXa7, a Type III Effector of Xanthomonas oryzae pv. Oryzae. Mol. Plant Microbe Interact., 18: 142-149. DOI: 10.1094/MPMI-18-0142

Zhu, W., B. Yang, J.M. Chittoor, L.B. Johnson and F.F. White, 1998. AvrXa10 contains an acidic transcriptional activation domain in the functionally conserved c terminus. Mol. Plant Microbe Interact., 11: 824-832. DOI: 10.1094/MPMI.1998.11.8.824 